\title{
SARCOMAS DE ALTO GRAU: ESTUDO RETROSPECTIVO DE 131 CASOS
}

\section{HIGH-GRADE SOFT TISSUE SARCOMAS: A RETROSPECTIVE ANALYSIS OF 131 CASES}

\author{
Wilmar José Manoel' ${ }^{1}$ Bruno José de Queiroz Sarmento²; Luiz de Paula Silveira Júnior; \\ Deidimar Cássia Batista de Abreu ${ }^{3}$; Iron Pires de Abreu Neto ${ }^{4}$; Erika Chaul Ferreira ${ }^{4}$
}

\begin{abstract}
RESUMO: Objetivo: Relatar as características clínicas dos sarcomas de partes moles de alto grau e apresentar a experiência do Hospital Araújo Jorge no tratamento destes sarcomas. Método: Análise retrospectiva dos casos de sarcoma de alto grau em adultos admitidos no Hospital Araújo Jorge (HAJ) entre 1996 e 2000. Idade, sexo, características anátomo-patológicas (tamanho e tipo histológico), localização, tratamentos oncológicos realizados (cirurgias de preservação de órgãos e membros, margens, quimioterapia, radioterapia), recorrência local, recorrência distante e sobrevida foram estudados. Análise descritiva, curvas de Kaplan-Meier, logrank test e teste $\div^{2}$ foram usados quando pertinentes. Resultados: Foram registrados 235 pacientes com sarcomas de partes moles entre 1996 - 2000, sendo que 131 eram de alto grau. A média de idade foi de 47,2 anos. O tipo histológico não foi determinado em $23,7 \%$ dos casos. O tipo mais freqüente foi o leiomiossarcoma $(13,7 \%)$, seguido do sarcoma sinovial $(10,7 \%)$ e rabdomiossarcoma $(9,2 \%)$. O tamanho mediano foi de $10 \mathrm{~cm}(2-48 \mathrm{~cm})$. A distribuição nos estádios II,III e IV foi de $15 \%$, 55\% e $30 \%$, respectivamente. Nos pacientes com estádios II e III, a margem cirúrgica adequada foi obtida em $51,9 \%$ dos pacientes. Radioterapia e quimioterapia adjuvantes foram indicadas em $33,7 \%$ e $26,1 \%$ dos casos, respectivamente. As recorrências locais e distantes ocorreram em $31,5 \%$ e $34,8 \%$ dos pacientes, respectivamente. A sobrevida global em 5 anos foi $61,8 \%$. Conclusão: A maioria dos pacientes atendidos no HAJ é portadora de lesões localmente avançadas, volumosas ou com metástase ao diagnóstico. Os pacientes apresentaram evolução adversa, com altas taxas de recorrência local e distante (Rev. Col. Bras. Cir. 2008; 35(2): 083-087).
\end{abstract}

Descritores: Neoplasias; Sarcoma; Cirurgia; Quimioterapia; Radioterapia.

\section{INTRODUÇÃO}

Os sarcomas de partes moles compõem um grupo heterogêneo de neoplasias malignas com diferentes padrões morfológicos da linhagem mesenquimal, representando cerca de $1 \%$ das neoplasias malignas em adultos. A maioria dos sarcomas de partes moles localiza-se nas extremidades, seguidos em ordem de freqüência pela cavidade abdominal, retroperitônio, tórax e cabeça e pescoço ${ }^{1,2}$.

A evolução dos sarcomas de partes moles pode ser estimada por alguns fatores prognósticos simplificados. $\mathrm{O}$ estadiamento preconizado pelo sistema TNM da União Internacional Contra o Câncer (UICC) utiliza o tamanho, a profundidade, o grau histológico e a presença de metástases linfonodais ou à distância para composição dos estádios ${ }^{3}$. Além destes fatores, a idade, o tipo histológico, a presença de margens comprometidas e tratamento cirúrgico realizado fora dos centros de referência parecem se associar com um pior prognóstico ${ }^{4,5,6}$

Os sarcomas de alto grau são caracterizados por biologia tumoral mais agressiva e um risco elevado de disseminação metastática. Ainda hoje, os sarcomas de alto grau representam um desafio ao tratamento oncológico multimodal, tanto em termos de preservação de órgãos e membros, como de redução do risco de metástase e morte.

Os objetivos deste trabalho são relatar as características clínicas dos sarcomas de partes moles de alto grau e apresentar a experiência do Hospital Araújo Jorge (HAJ) no tratamento destes sarcomas. clínica.

\section{MÉTODO}

Trata-se de um estudo retrospectivo descritivo envolvendo pacientes com diagnóstico histológico de sarcoma de partes moles de alto grau admitidos no Hospital Araújo Jorge entre 1996 e 2000. Foram incluídos pacientes acima de 18 anos, em todos os estádios e localizações.

Os dados foram extraídos do banco de dados de sarcomas do Serviço de Tecido Conjuntivo do HAJ. Registramos as informações referentes à idade, ao sexo, às características anátomo-patológicas (tamanho e tipo histológico), ao tipo de operação (preservação de órgãos e membros ou mutiladora), às margens cirúrgicas (adequadas ou inadequadas), à quimioterapia e à radioterapia. Foram incluídos neste estudo os pacientes com laudos de imunohistoquímica (IHQ). Também foram incluídos aqueles pacientes em que a análise

1. Chefe do Serviço de Tecido Conjuntivo do Hospital Araújo Jorge - Goiânia -GO

2. Cirurgião Oncológico do Hospital Regional de Taguatinga - Secretaria de Estado de Saúde do Distrito Federal - Brasília. Membro Titular da Sociedade Brasileira de Cirurgia Oncológica.

3. Membro Titular do Serviço de Tecido Conjuntivo do Hospital Araújo Jorge-Goiânia.

4. Acadêmico de Medicina da Universidade Federal de Goiás-Goiânia.

Recebido em 19/10/2007

Aceito para publicação em 20/12/2007

Conflito de interesses: nenhum

Fonte de financiamento: nenhuma

Trabalho realizado no Serviço de Tecido Conjuntivo, Hospital Araújo Jorge, Associação de Combate ao Câncer em Goiás, Goiânia-GO 
imunohistoquímica não foi realizada ou foi inconclusiva, desde que fossem consistentemente compatíveis com sarcoma fusocelular. De acordo com a diferenciação, número de mitoses e necrose, os tumores foram classificados com um sistema de três graus. Seguindo a orientação da $\mathrm{UICC}^{3}$, os tumores Grau I foram denominados de baixo grau e os tumores Graus II e III, reclassificados como de alto grau. As margens de ressecção foram consideradas adequadas se livres de neoplasia e com mais de $1 \mathrm{~cm}$ e classificamos como margens inadequadas ressecções intralesionais, marginais, com margem comprometida ou ruptura do tumor. Consideramos como operação mutiladora quando havia o relato de amputação de algum membro ou a ressecção de órgãos em monobloco e cirurgia de preservação quando havia o relato de excisão local ampla e sem ressecções de órgãos adjacentes ou amputação. Os sarcomas de extremidades, parede torácica e parede abdominal foram definidos como grupo de localização favorável e os sarcomas de cabeça/pescoço, pélvicos, viscerais e retroperitoniais foram denominados de grupo desfavorável. Para análise dos resultados do tratamento com intenção curativa excluímos pacientes no estádio IV e portadores de sarcoma de Kaposi.

O estadiamento obedeceu à versão TNM 2002 da UICC. Para pacientes com sarcomas extra-abdominais utilizamos tomografia computadorizada (TC) de tórax e ultra-som (US) abdominal. Os portadores de sarcoma intra-abdominal foram avaliados com TC de tórax e abdômen. O tumor primário foi avaliado inicialmente com TC e a ressonância magnética quando se julgou necessária para programação cirúrgica. $\mathrm{O}$ seguimento foi realizado com exame clínico do sítio operado, radiografia de tórax e US de abdômen a cada três meses nos primeiros dois anos e depois semestralmente até completar cinco anos de tratamento.

A análise estatística consistiu num estudo descritivo, curvas de sobrevida de Kaplan-Meier, log-rank test para diferenças entre as curvas, cálculo da sobrevida mediana e teste $\div^{2}$, quando pertinentes. Os dados foram analisados com o software SPSS 10.0, EUA. Valores de $\mathrm{p}<0,05$ serão considerados significativos. Os pacientes assinaram o termo de consentimento informado padronizado pelo Hospital Araújo Jorge antes de serem submetidos ao tratamento cirúrgico, à quimioterapia ou à radioterapia. $\mathrm{O}$ estudo foi aprovado pelo Comitê de Ética da Associação de Combate ao Câncer em Goiás.

\section{RESULTADOS}

Durante o período de 1996 e 2000 foram admitidos 215 pacientes adultos com diagnóstico de sarcomas de partes moles. Os tumores de alto grau somaram 131 pacientes $(60,9 \%)$, $84(39,1 \%)$ foram classificados como sarcomas de baixo grau. As características gerais dos pacientes são demonstradas na Tabela 1. Verificamos que a maioria dos pacientes era do sexo masculino (53,4\%). A idade mediana foi de 48 anos. O sítio mais freqüente foi o membro inferior $(37,4 \%)$. O tamanho mediano do tumor foi de $10 \mathrm{~cm}$ variando de $2 \mathrm{~cm}$ a $48 \mathrm{~cm}$. As metástases distantes estavam presentes ao diagnóstico em 32 pacientes $(24,4 \%)$ e os linfonodos apresentaram-se com metástase em 11 pacientes $(8,4 \%)$. Os pacientes com sarcomas de alto grau foram distribuídos em estádios nas seguintes proporções: 20 no estádio II (15,3\%), 72 no estádio III (55\%) e 39 no estádio IV $(29,8 \%)$. Trinta e um pacientes $(27,7 \%)$ não tiveram a histogênese definida, sendo tratados como portadores de sarcoma fusocelular sem outras especificações.

A Tabela 2 mostra as modalidades de tratamentos empregados no HAJ. A radioterapia neoadjuvante foi utilizada em $4,3 \%$ e a quimioterapia neoadjuvante em $6,5 \%$ dos paci-

Tabela 1 - Características dos 131 pacientes com sarcomas de alto grau.

\begin{tabular}{|c|c|c|}
\hline \multirow{2}{*}{$\begin{array}{l}\text { Características } \\
\text { Sexo }\end{array}$} & \multicolumn{2}{|c|}{ Pacientes $(\%)$} \\
\hline & & \\
\hline Masculino & 70 & $(53,4)$ \\
\hline Feminino & 61 & $(46,6)$ \\
\hline Idade mediana (variação) & 48 anos & $(18-87)$ \\
\hline \multicolumn{3}{|l|}{ Localização } \\
\hline Membro inferior & 49 & $(37,4)$ \\
\hline Visceral & 20 & $(15,3)$ \\
\hline Retroperitônio & 16 & $(12,2)$ \\
\hline Pelve & 12 & $(9,2)$ \\
\hline Parede torácica & 12 & $(9,2)$ \\
\hline Membro superior & 12 & $(9,2)$ \\
\hline Cabeça/Pescoço & 9 & $(6,9)$ \\
\hline Parede abdominal & 1 & $(0,8)$ \\
\hline Mestástases & 32 & $(24,4)$ \\
\hline Linfonodos comprometidos & 11 & $(8,4)$ \\
\hline \multicolumn{3}{|l|}{ Estadio } \\
\hline II & 20 & $(15,3)$ \\
\hline III & 72 & $(55)$ \\
\hline IV & 39 & $(29,8)$ \\
\hline \multicolumn{3}{|l|}{ Tipo histológico } \\
\hline Sarcoma fusocelular & 31 & $(27,7)$ \\
\hline Leiomiossarcoma & 18 & $(13,7)$ \\
\hline Sarcoma sinovial & 14 & $(10,7)$ \\
\hline Rabdomiossarcoma & 12 & $(9,2)$ \\
\hline Fibrossarcoma & 11 & $(8,4)$ \\
\hline Neurossarcoma & 10 & $(7,6)$ \\
\hline Lipossarcoma & 8 & $(6,1)$ \\
\hline Angiossarcoma & 8 & $(6,1)$ \\
\hline Outros & 19 & $(14,5)$ \\
\hline
\end{tabular}

Tabela 2 - Tipos de tratamentos utilizados nos 92 pacientes com sarcomas de alto grau nos estadios II e III.

\begin{tabular}{lcc}
\hline Tipo & \multicolumn{2}{c}{ Freqüência $(\%)$} \\
\hline RT neoadjuvante & 4 & $(4,3)$ \\
QT neoadjuvante & 6 & $(6,5)$ \\
RT adjuvante & 31 & $(33,7)$ \\
QT adjuvante & 24 & $(26,1)$ \\
QT com antracíclicos/ifosfamida & 18 & $(19,5)$ \\
Cirurgia de preservação & 49 & $(53,3)$ \\
Margens inadequadas/não avaliáveis & 30 & $(32,6)$ \\
\hline
\end{tabular}


entes. As cirurgias de preservação de órgãos ou membros foram realizadas em 53,3\% dos casos. As margens foram inadequadas em 53,3\% das peças, obtendo-se cirurgia R0 em $78 \%$ (39/50) das lesões com localização favorável, enquanto no grupo desfavorável a cirurgia R0 foi registrada em 45,7\% $(16 / 35)$ dos casos $(\mathrm{p}=0,002)$. A quimioterapia foi utilizada em 24 pacientes na adjuvância $(26,1 \%)$, com esquemas contendo doxorrubicina ou epirrubicina associadas à ifosfamida em 18 pacientes $(19,5 \%)$. A radioterapia adjuvante foi indicada em 31 pacientes $(33,7 \%)$.

A recorrência local foi verificada 29 pacientes $(31,5 \%)$ e recorrência distante em 32 pacientes $(34,8 \%)$. A recorrência local ocorreu nos grupos de localização favorável e desfavorável em $33,9 \%$ e $28,2 \%$, respectivamente $(\mathrm{p}=0,55)$. Os pacientes com margens inadequadas apresentaram 43,3\% de recorrência local e no grupo com margens adequadas a recorrência local foi de $29 \%$ ( $\mathrm{p}=0,18$ ). De forma global, 48 pacientes $(52,2 \%)$ apresentaram algum tipo de recorrência isolada ou combinada, levando a uma sobrevida livre de recorrência em cinco anos de 19,5\% (Tabela 3). Cinqüenta e oito pacientes $(40,2 \%)$ foram admitidos após terem sido submetidos a procedimentos cirúrgicos em instituições não especializadas em tratamento oncológico.

A Figura 1 mostra que a sobrevida global em cinco anos foi de $94 \%$ para sarcomas de baixo grau e $61,8 \%$ para os sarcomas de alto grau ( $\mathrm{p}=0,0001)$. Nos pacientes com sarcomas de alto grau, a sobrevida mediana foi de 16,5 meses, a sobrevida global em cinco anos no estádio II foi $69,3 \%$, no estádio III de $64,4 \%$ e no estádio IV foi de $47,7 \%$, sem diferença estatística (Figura 2).

Os grupos que se submeteram à quimioterapia ou radioterapia adjuvantes não são homogêneos (dados não mostrados). A sobrevida em cinco anos para os pacientes que receberam quimioterapia adjuvante e que não receberam foi de $56,9 \%$ e $61,7 \%$ ( $p=0,70)$, respectivamente. Os pacientes que se submeteram à radioterapia adjuvante obtiveram sobrevida global em cinco anos de 72,8\% contra 59,2\% no Grupo tratado sem radioterapia $(p=0,89)$. Podemos verificar uma tendência de pior sobrevida em cinco anos para sarcomas de localização desfavorável $(59,2 \%)$ comparada com a SG dos sarcomas favoráveis $(69,3 \%)$, com $\mathrm{p}=0,06$ (Figura 3 ).

\section{DISCUSSÃO}

Nesta série, chama a atenção um índice de recidiva local em sarcomas de localização favorável de 28,2\%, atual-

Tabela 3 - Resultados do tratamento dos 92 pacientes com sarcomas de alto grau nos estadios II e III.

\begin{tabular}{ll}
\hline Objetivos & Resultado \\
\hline Recorrência local & $29 \quad(31,5 \%)$ \\
Recorrência metastática & $32 \quad(34,8 \%)$ \\
Sobrevida mediana livre de recorrência & 11 meses \\
Sobrevida livre de recorrência em 5 anos & $19,5 \%$ \\
Sobrevida mediana & 16,5 meses \\
Sobrevida global em 5 anos & $61,8 \%$ \\
\hline
\end{tabular}

mente considerada elevada. De forma geral, estes resultados podem ser atribuídos a indicação pouco frequiente de radioterapia adjuvante, ao estádio avançado das neoplasias à admissão, bem como à alta proporção de pacientes que foram submetidos a procedimentos invasivos fora dos centros de referência. Com relação aos sarcomas de extremidades, o objetivo

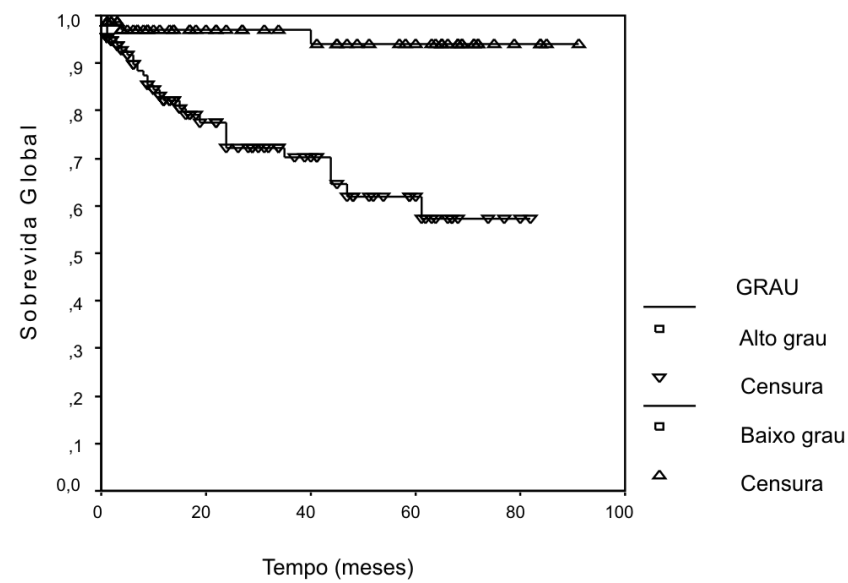

Figura 1 - Curva de sobrevida global dos pacientes com sarcomas de partes moles tratados no HAJ de acordo com o grau histológico.

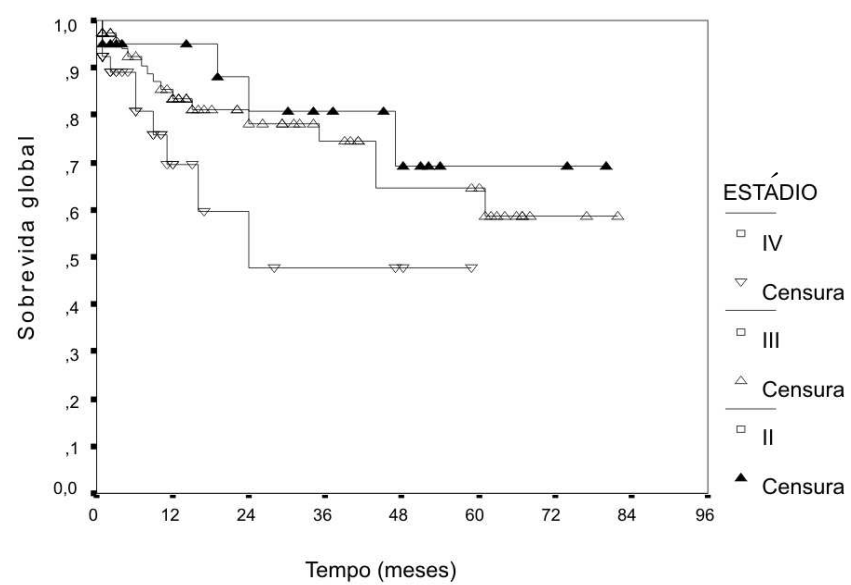

Figura 2 - Curva de sobrevida global dos pacientes com sarcomas de alto grau de acordo com o estadio TNM 2002.

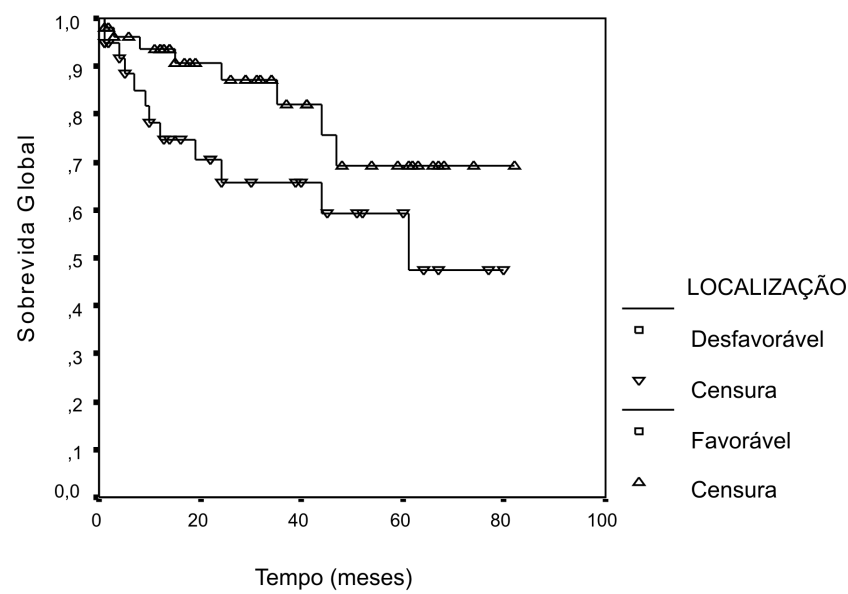

Figura 3 - Curvas de sobrevida global dos pacientes com sarcomas de alto grau de acordo com a abordagem da lesão (favorável $x$ desfavorável). 
de preservar o membro e obter controle local satisfatório é estudado em três linhas principais. A primeira linha de pesquisa avalia as cirurgias de preservação de membros e radioterapia adjuvante com dose total de $60 \mathrm{~Gy}$. Os resultados obtidos mostram que a recorrência local pode ocorrer entre $8 \%$ e $24 \%$ dos pacientes e que não há incremento na sobrevida global com a amputação do membro em todos os pacientes de forma sistemática ${ }^{7,8,9,10}$. A segunda linha é representada pela radioterapia neoadjuvante com quimioterapia sistêmica associada. No Massachusetts General Hospital, associando-se o programa MAID (mesna, adriamicina, ifosfamida e dacarbazina) e radioterapia neoadjuvante com $44 \mathrm{~Gy}$, o controle local em cinco anos foi de $92 \%{ }^{11}$. O grupo do Roswell Park Cancer Institute também utilizou um programa semelhante e obteve falência locorregional de $17,6 \%$ em três anos ${ }^{12}$. Uma série canadense empregando o protocolo de Eilber modificado mais 30 Gy de irradiação neoadjuvante registrou uma taxa de recidiva local de $3,8 \%$ num seguimento mediano de 6,1 anos $^{13}$. O avanço mais recente se deve a perfusão hipertérmica isolada do membro com melfalan e fator de necrose tumoral-alfa desenvolvida na Holanda. Esta técnica tem mostrado uma taxa de resposta de $88 \%$, resposta patológica completa de $42 \%$ e permitiu a preservação de membro em $82 \%$ dos casos com indicação de amputação ${ }^{14}$. Nos tumores retroperitoniais, a sobrevida mantém-se praticamente inalterada e o controle local persiste ruim mesmo com a utilização da radioterapia intraoperatória, com taxas que se situam entre 40 e $70 \%{ }^{15,16,17}$. Estes tratamentos locais não estão associados primariamente com a melhora da sobrevida global.

Outro ponto de controvérsia se refere às estratégias de redução da disseminação metastática e conseqüente melhora da sobrevida. Neste estudo, a recorrência distante ocorreu em 32 pacientes $(34,8 \%)$ e a sobrevida global em cinco anos foi de apenas $61,8 \%$. As melhores evidências para indicação de quimioterapia ainda se resumem a dois estudos. $\mathrm{Na}$ análise do Sarcoma Meta-analysis Collaboration publicada em 1997, incluindo sarcomas de diversas localizações, as taxas de recorrência local e distante foram $17,4 \%$ e $31,4 \%$, respectivamente. $\mathrm{O}$ benefício da quimioterapia com doxorrubicina foi demonstrado em termos de redução da recorrência local (hazard ratio $0,73, \mathrm{p}=0,016$ ) e da recorrência distante (hazard ratio $0,70, \mathrm{p}=0,0003$ ), porém apenas uma tendência à melhora da sobrevida foi verificada (hazard ratio $0,89, \mathrm{p}=0,12$ ). A análise do subgrupo de sarcomas de alto grau localizados nas extremidades evidenciou uma redução do risco de morte de $20 \%(\mathrm{p}=0,029)$, tornando a adjuvância baseada em doxorrubicina uma abordagem promissora para esta situação específica $^{18}$. A outra evidência é o estudo randomizado italiano publicado em 2001, no qual a quimioterapia sistêmica com epirrubicina e ifosfamida incrementou a sobrevida global em 4 anos em 19\% (69\% versus 50\%, p=0,04), contribuindo para maior aceitação da quimioterapia adjuvante para sarcomas de partes moles de alto grau com mais de $5 \mathrm{~cm}$, localizados nos membros ${ }^{19}$. Os resultados da quimioterapia adjuvante têm sido difíceis de ser reproduzidos e corroborados devido aos resultados poucos expressivos obtidos com as drogas atualmente disponíveis, a raridade da neoplasia e a heterogeneidade histológica habitual nos estudos de sarcomas.

A biologia tumoral e a evolução dos sarcomas ainda são apenas parcialmente conhecidas. O conhecimento dos inúmeros eventos genéticos e moleculares envolvidos ainda necessita de maiores avanços com objetivo de orientar melhor a definição da histogênese, determinar precisamente o prognóstico e desenvolver terapias-alvo, a exemplo do que tem ocorrido com os tumores estromais gastrintestinais (GIST's).

Os resultados obtidos neste trabalho demonstram que a maioria dos pacientes admitida no HAJ é portadora de lesões avançadas, com grandes massas tumorais ou doença disseminada.. Apesar das limitações do trabalho, fica evidente que os sarcomas de alto grau mesmo tratados com intenção curativa apresentam evolução adversa, caracterizada por alta freqüência de recorrência local e, principalmente, disseminação metastática, como descrita na literatura.

\begin{abstract}
Background: To describe clinical presentation of high-grade soft tissue sarcomas, oncologic management and results at Araújo Jorge Hospital (HAJ). Methods: We analyzed 131 patients with high-grade soft sarcomas in adults admitted at Araújo Jorge Hospital between 1996 and 2000. We studied, age, gender, pathological characteristics (size and histological type), localization, oncology treatment (organ or limb-sparing approach, margins, chemotherapy, and radiotherapy), local recurrence, distant recurrence and overall survival. We used descriptive analysis, Kaplan-Meier methodology, log-rank test and Chi-square test. Results: Two hundred thirty-five patients with soft tissue sarcomas were admitted between 1996 and 2000. A hundred thirty-one were high-grade sarcomas. Median age was 47.2 years. We could not determinate a histological type in $23.7 \%$ of cases. The more frequents types were leiomyosarcoma (13.7\%), synovial sarcoma (10.7\%) and rhabdomyosarcoma (9.2\%). Median size was $10 \mathrm{~cm}(2-48 \mathrm{~cm})$. The distribution for stages II, III e IV was $15 \%$, 55\% and $30 \%$, respectively. For stages II and III, surgical margins were adequate in $51.9 \%$ of cases. Radiotherapy and chemotherapy were performed in $33.7 \%$ and $26.1 \%$ of cases, respectively. Local recurrences occurred in $31.5 \%$ and distant recurrences in $34.8 \%$ of patients. The 5-year overall survival was 61.8\%. Conclusion: Most of the patients that were treated at Araújo Jorge Hospital have large and locally advanced tumors, with metastatic spread at first presentation. The patients with highgrade sarcomas presented adverse clinical evolution, high rates of local and distant recurrences as described in the literature.
\end{abstract}

Key words: Neoplasms; Sarcoma; Surgery; Drug therapy; Radiotherapy. 


\section{REFERÊNCIAS}

1. Singer S, Demetri GD, Baldini EH, Fletcher CD. Management of soft-tissue sarcomas: an overview and update. Lancet Oncol. $2000 ; 1: 75-85$.

2. Latorre MR, Franco EL. Epidemiologia dos sarcomas. In: Lopes A. Sarcomas de partes moles. Rio de Janeiro: Medsi; 1999. p. 3-18.

3. International Union Against Cancer (UICC). TNM: classification of malignant tumors. $6^{\text {th }}$ ed. New York: Wiley-Liss; 2002.

4. Coindre JM, Terrier P, Bui NB, Bonichon F, Collin F, Le Doussal $\mathrm{V}$, et al. Prognostic factors in adult patients with locally controlled soft tissue sarcoma. A study of 546 patients from the French Federation of Cancer Centers Sarcoma Group. J Clin Oncol. 1996; 14(3):869-77.

5. Stojadinovic A, Leung DH, Allen P, Lewis JJ, Jaques DP, Brennan MF. Primary adult soft tissue sarcoma: time-dependent influence of prognostic variables. J Clin Oncol. 2002; 20(21):4344-52.

6. Alektiar KM, Brennan MF, Singer S. Influence of site on the therapeutic ratio of adjuvant radiotherapy in soft-tissue sarcoma of the extremity. Int J Radiat Oncol Biol Phys. 2005; 63(1):202-8

7. Rosenberg SA, Tepper J, Glatstein E, Costa J, Baker A, Brennan M, DeMoss EV, Seipp C, Sindelar WF, Sugarbaker P, Wesley R. The treatment of soft-tissue sarcomas of the extremities: prospective randomized evaluations of (1) limb-sparing surgery plus radiation therapy compared with amputation and (2) the role of adjuvant chemotherapy. Ann Surg. 1982; 196(3):305-15.

8. Keus RB, Rutgers EJ, Ho GH, Gortzak E, Albus-Lutter CE, Hart AA. Limb-sparing therapy of extremity soft tissue sarcomas: treatment outcome and long-term functional results. Eur J Cancer. 1994; 30A(10):1459-63.

9. Williard WC, Hajdu SI, Casper ES, Brennan MF. Comparison of amputation with limb-sparing operations for adult soft tissue sarcoma of the extremity. Ann Surg. 1992; 215(3):269-75.

10. Karakousis CP, Zografos GC. Radiation therapy for high grade soft tissue sarcomas of the extremities treated with limbpreserving surgery. Eur J Surg Oncol. 2002; 28(4):431-6.

11. DeLaney TF, Spiro IJ, Suit HD, Gebhardt MC, Hornicek FJ, Mankin HJ, Rosenberg AL, Rosenthal DI, Miryousefi F, Ancukiewicz M, Harmon DC. Neoadjuvant chemotherapy and radiotherapy for large extremity soft-tissuesarcomas. Int J Radiat Oncol Biol Phys. 2003; 56(4):1117-27.

12. Kraybill WG, Harris J, Spiro IJ, Ettinger DS, DeLaney TF, Blum RH, Lucas DR, Harmon Dc, Letson GD, Eisenberg B; Radiation Therapy Oncology Group Trial 9514. Phase II study of neoadjuvant chemotherapy and radiation therapy in the management of high-risk, high-grade, soft tissue sarcomas of the extremities and body wall. J Clin Oncol. 2006; 24(4):619-25.
13. Temple CL, Ross DC, Magi E, DiFrancesco LM, Kurien E, Temple WJ. Preoperative chemoradiation and flap reconstruction provide high local control and low wound complication rates for patients undergoing limb salvage surgery for upper extremity tumors. J Surg Oncol. 2007; 95(2):135-41.

14. Grunhagen DJ, Brunstein F, Graveland WJ, van Geel AN, de Wilt JH, Eggermont AM. Isolated limb perfusion with tumor necrosis factor and melphalan prevents amputation in patients with multiple sarcomas in arm or leg. Ann Surg Oncol. 2005; 12(6):473-9. Epub 2005 Apr 19.

15. Alektiar KM, Hu K, Anderson L, Brennan MF, Harrison LB High-dose-rate intraoperative radiation therapy (HDR-IORT) for retroperitoneal sarcomas. Int J Radiat Oncol Biol Phys. 2000; 47(1):157-63.

16. Ballo MT, Zagars GK, Pollock RE, Benjamin RS, Feig BW, Cormier JN, et al. Retroperitoneal soft tissue sarcoma: an analysis of radiation and surgical treatment. Int J Radiat Oncol Biol Phys. 2007; 67(1):158-63. Epub 2006 Nov 2.

17. Youssef E, Fontanesi J, Mott M, Kraut M, Lucas D, Mekhael $\mathrm{H}$, et al. Long-term outcome of combined modality therapy in retroperitoneal and deep-trunk soft-tissue sarcoma: analysis of prognostic factors. Int J Radiat Oncol Biol Phys. 2002; 54(2):514-9.

18. Adjuvant chemotherapy for localised resectable soft-tissue sarcoma of adults: meta-analysis of individual data. Sarcoma Metaanalysis Collaboration. Lancet. 1997; 350(9092):1647-54.

19. Frustaci S, Gherlinzoni F, de Paoli A, Bonetti M, Azzarelli A, Comandone A, et al. Adjuvant chemotherapy for adult soft tissue sarcomas of the extremities and girdles: results of the Italian randomized cooperative trial. J Clin Oncol. 2001; 19(5):1238-47.

Como citar este artigo:

Manoel WJ, Sarmento BJ, Silveira Jr. LP, Abreu DC, Abreu Neto IP, Ferreira EC

Sarcomas de alto grau: estudo retrospectivo de 131 casos. Rev Col Bras Cir. [periódico na Internet] 2008; 35(2). Disponível em URL: http://www.scielo.br/rcbc

Endereço para correspondência:

Wilmar José Manoel

Rua S6, 664

Rua $239, n^{\circ} 206$.

Setor Bela Vista

74823-470 - Goiânia - GO

Fone: (62) 3255-1482

E-mail:wilmarmanoel@brturbo.com.br 